\title{
Desplazamiento de las fracturas de ángulo mandibular y su relación con el tercer molar según la clasificación de Pell y Gregory
}

\author{
Displacement of mandibular angle fractures and their relationship to \\ the third molar according to the Pell and Gregory classification
}

Luis Felipe Reyes Chinchilla,* Servio Interiano, ${ }^{+}$Guillermo Barreda, ${ }^{\S}$ Jaime Matta ${ }^{\mathbb{I}}$

\section{RESUMEN}

El trauma facial es una de las causas más comunes por la cual se consulta al Departamento de Estomatología en el área de Cirugía Oral y Maxilofacial del Hospital Roosevelt. El objetivo de este estudio fue determinar el desplazamiento de las fracturas de ángulo mandibular y su relación con la presencia del tercer molar inferior en trazo de fractura según la clasificación de Pell y Gregory. Material y métodos: Se diseñó un estudio observacional transversal en donde se evaluaron todos los pacientes que ingresaron a emergencias del Hospital Roosevelt en el área de Cirugía Oral y Maxilofacial, se seleccionaron las historias clínicas que incluyeran la edad, pacientes con fracturas de ángulo mandibular con los terceros molares incluidos en trazo de fracturas, que contaran con tomografía asistida por computador (TAC) y sin la presencia de otras fracturas faciales. Resultados: Análisis de datos de un total de 45 pacientes; se observó que la posición más frecuente del tercer molar según la clasificación de

\section{ABSTRACT}

Facial trauma is one of the most common causes for which the Stomatology Department is consulted in the area of Oral and Maxillofacial Surgery at Roosevelt Hospital. Objective of this study was to determine the displacement of mandibular angle fractures and its relationship with the presence of the lower third molar in the fracture line according to the Pell and Gregory classification. Material and methods: A cross-sectional observational study was designed in which all patients admitted to the Roosevelt Hospital emergency in the area of Oral and Maxillofacial Surgery were evaluated, the medical records that included their data such as age, patients with mandibular angle fractures with the third molars included in the fracture trace, which will have computer assisted tomography (CT), without the presence of other facial fractures. Results: Data analysis of a total of 45 patients, it was observed that the most frequent position of the third molar according to the Pell and Gregory classification

* Médico residente de IV año de la Maestría en Cirugía Oral de la Universidad de San Carlos de Guatemala. Hospital Roosevelt, Guatemala.

₹ Cirujano Dentista, Maestría de Estadística de la Universidad de San Carlos de Guatemala.

$\S$ Decano de la Facultad de Odontología de la Universidad de San Carlos de Guatemala.

" Jefe del Departamento de Estomatología del Hospital Roosevelt, Guatemala.

Correspondencia:

Luis Felipe Reyes Chinchilla

E-mail: luisferechi89@gmail.com

Citar como: Reyes CLF, Interiano S, Barreda G, Matta J. Desplazamiento de las fracturas de ángulo mandibular y su relación con el tercer molar según la clasificación de Pell y Gregory. Rev Mex Cir Bucal Maxilofac. 2021;17 (1): 4-10. https://dx.doi.org/10.35366/100254 
Pell y Gregory fue Al con un $31.10 \%$. Conclusión: Se analizó el comportamiento de las fracturas de ángulo mandibular en relación con la posición del tercer molar; su desplazamiento no está condicionado por la presencia de ésta en trazo de fractura, por lo que se descarta que el tercer molar juegue un papel importante que permita o no el desplazamiento de la fractura de ángulo mandibular, ya sea favorable o desfavorable en los 45 casos estudiados.

Palabras clave: Fractura mandibular, fracturas de ángulo mandibular, tercer molar en trazo de fractura, fracturas faciales, trauma mandibular, tercer molar incluido. was Al with $31.10 \%$. Conclusion: It was observed that the behavior of mandibular angle fractures in relation to the position of the third molar and its displacement is not conditioned by the presence of the fracture line, so it is ruled out that the presence of the third molar plays a role. an important role that allows or not the displacement of the mandibular angle fracture, whether favorable or unfavorable in the 45 cases studied.

Keywords: Mandibular fracture, mandibular angle fractures, third molar in fracture line, facial fractures, mandibular trauma, third molar included.

\section{INTRODUCCIÓN}

La presente investigación se refiere al tema de desplazamiento de las fracturas de ángulo mandibular y su relación con la posición según la clasificación de Pell y Gregory de los terceros molares en pacientes atendidos en el Departamento de Estomatología del Hospital Roosevelt, en donde la incidencia de las fracturas faciales es alta, en su mayoría causadas por accidentes viales (accidentes en motocicleta). Las fracturas faciales que con mayor frecuencia se observaron y que coinciden con la literatura son las de ángulo mandibular, siendo una predisposición a fractura la presencia del tercer molar, pudiendo descubrir una fractura favorable (la acción muscular acerca los fragmentos) o desfavorables (la acción muscular separa los fragmentos).

Se define como fractura de ángulo a la discontinuidad ósea localizada detrás del segundo molar inferior, en el punto en el que se unen el cuerpo y la rama ascendente mandibular. ${ }^{2}$

La investigación se basa en el interés sobre la relación entre estos elementos, la posición que tiene el tercer molar y la dirección de la fractura.

Se realizó la evaluación de la tomografía asistida por computador en cortes coronales y sagitales en todos los pacientes que cumplieran los criterios de inclusión: tercer molar en trazo de fractura, mayores de 15 años y sin fracturas dobles mandibulares 0 de tercio medio facial; tomando como referencia el conducto dentario inferior para medir la distancia entre los segmentos fracturados y evaluar la posición del tercer molar, con el objetivo de determinar el desplazamiento de las fracturas de ángulo mandibular y su relación con la presencia del tercer molar inferior en trazo de fractura, según la clasificación de Pell y Gregory. ${ }^{3}$

\section{MATERIAL Y MÉTODOS}

Se diseñó un estudio observacional transversal en donde se evaluó a los pacientes que ingresaron a la emergencia del Hospital Roosevelt en el Departamento de Estomatología, Cirugía Oral y Maxilofacial en Guatemala, el que presentó fracturas faciales con las características de las fracturas de ángulo mandibular con los terceros molares incluidos en trazo de fracturas en el periodo de enero a noviembre de 2019, siendo un total de 45 casos que cumplieron los criterios de inclusión. Su trauma facial fue causado por accidente en motocicleta y todos fueron del sexo masculino sin casos reportados del sexo femenino.

\section{Plan de análisis}

Los datos obtenidos en la evaluación de las tomografías computarizadas con cortes coronales y sagitales fueron vaciados en una hoja electrónica del programa Epi Info versión 6.01. Los resultados obtenidos fueron analizados con estadística descriptiva, presentándolos en tablas para cada una de las variables estudiadas, indicando la frecuencia y el porcentaje de incidencia de cada una de ellas. De igual forma se presentaron los gráficos de barras cuando se estimó conveniente, teniendo como parámetros anatómicos el techo del conducto dentario inferior como una forma para medir la distancia de los fragmentos óseos vistos en la tomografía computarizada (Tabla 1 y Figura 1).

\section{RESULTADOS}

En el periodo comprendido de enero a noviembre del 2018 se evaluaron 45 pacientes que cumplieron los criterios de inclusión antes mencionados.

Se registra que las edades más frecuentes en las fracturas de ángulo mandibular con tercer molar en 
trazo de fractura están en el rango comprendido de los 17 a 32 años con un porcentaje de 35 a 13.33\% y una menor tendencia en las edades comprendidas entre los rangos de 37 a 47 años con el 6.67 a $2.22 \%$ de frecuencia, con una media de 26.51 años y una desviación estándar de 6.8 (Figura 2). La desviación cuartil es de 4, lo que indica la diferencia entre cada intervalo de cuatro años.

Se observó que los terceros molares en la posición Al fueron más frecuentes en las fracturas de ángulo mandibular con un $31.10 \%$, en segundo lugar fueron las piezas en la clasificación BIII con un $20 \%$, en tercer lugar las piezas en la clasificación All y con un porcentaje significativo del $15 \%$ las que están en la clasificación de CIII, siendo por lo tanto menos frecuentes las demás clasificaciones (Figura 3).

Se registró que la mayor frecuencia de desfavorabilidad de las fracturas por su grado de desplaza-

Tabla 1: Evaluación de la relación entre las variables de desplazamiento, posición y clase del tercer molar según la clasificación de Pell y Gregory.

\begin{tabular}{|c|c|c|c|}
\hline \multirow[b]{2}{*}{ Desplazamiento (mm) } & \multicolumn{3}{|c|}{ Posición y clase } \\
\hline & Al, II y III & BI, II y III & $\mathrm{Cl}$, II y III \\
\hline \multicolumn{4}{|l|}{$>6$} \\
\hline \multicolumn{4}{|l|}{$5-6$} \\
\hline \multicolumn{4}{|l|}{$1-2$} \\
\hline \multicolumn{4}{|l|}{$3-4$} \\
\hline 0 & & & \\
\hline
\end{tabular}

Muy favorable: $0 \mathrm{~mm}$ de desplazamiento; Medio favorable: 3-4 mm de desplazamiento; Poco favorable: 1-2 mm de desplazamiento; Desfavorable: 5-6 mm de desplazamiento; Muy desfavorable: $>6 \mathrm{~mm}$ de desplazamiento.

Fuente: Elaboración propia (2019). miento es de $33 \%$, lo que significa muy desfavorable, por ser mayor de $6 \mathrm{~mm}$. Con menor frecuencia fue muy favorable el $7 \%$ que es igual a un desplazamiento de $0 \mathrm{~mm}$.

Para medir el grado de desplazamiento, se tomó como punto anatómico de referencia el techo del conducto del paquete neurovascular dentario inferior con el uso de la tomografía asistida por computador en sus cortes coronales y sagitales (Figura 4).

Se observó que es mayor la frecuencia de la posición del tercer molar Al con relación en las fracturas de ángulo mandibular. Se registró que la mayor frecuencia de desfavorabilidad es de 3 (poco favorable: 1-2 mm de desplazamiento).

Correlacionando ambas variables, vemos que no existe una relación íntima entre la posición que tenga el tercer molar, presente en el trazo de fractura, por no existir una constante entre la posición y el grado de desplazamiento (Tabla 2).

Se registró que la edad más frecuente es de 24 años, la cual no tiene relación con la desfavorabilidad, que es de 3 (Tabla 3).

\section{DISCUSIÓN}

Entre los factores etiológicos más comunes de las fracturas faciales se observan los accidentes viales, la práctica de deportes, la violencia y los traumatismos casuales, siendo los hombres los expuestos a estos accidentes. ${ }^{2}$ La reposición de los fragmentos de la fractura podría ser más rápida y fácil, si el diente en la línea de fractura se maneja conservadoramente. El diente podría proveer una referencia oclusal y un tope posterior. Esto tendría un efecto de estabilización y no permitiría que el hueso se desplazara. Si se extrajera, esto incrementaría el riesgo de
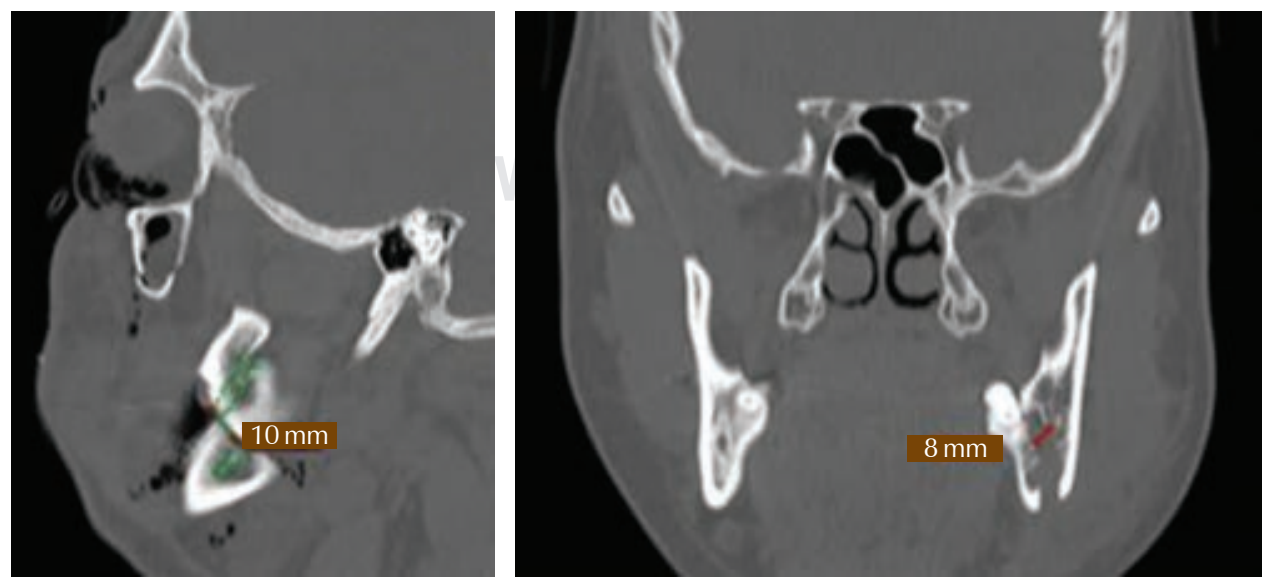

Figura 1:

Distancia de los segmentos óseos en corte sagital y coronal, tomando como punto de referencia el techo del conducto dentario inferior. Fuente: Captura de pantalla de una tomografía asistida por computador en cortes sagital y coronal (2018). 


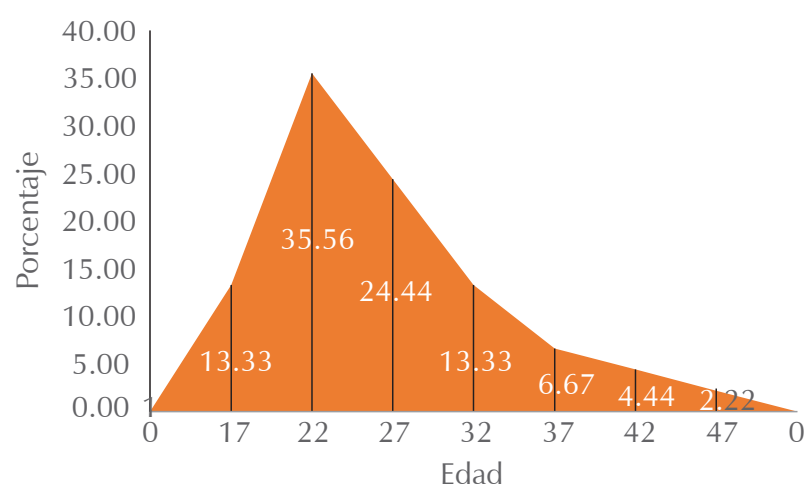

Figura 2: Porcentaje más frecuente en la edad de los pacientes incluidos en la muestra.

Fuente: Trabajo de campo elaborado con Microsoft Excel (2017).

que la fractura se contaminara y en algunos casos podría dificultar la sutura. ${ }^{1}$ En la revisión de la literatura se mostró la controversia de conservar o no el tercer molar en trazo de fractura; en el estudio realizado se observó que no siempre se amerita su extracción, así también que éste puede llegar a ser un causal de complicaciones postoperatorias. Por otra parte, se carece de literatura que evidencie la relación del grado de desplazamiento de la fractura con la posición que tenga el tercer molar inferior. En los resultados obtenidos en nuestra muestra se observó que los terceros molares, según la clasificación de Pell y Gregory ${ }^{3}$ en la posición «Al», fueron más frecuentes en las fracturas de ángulo mandibular con un 30\%. Teniendo como referencia la literatura existente, los terceros molares en posición BIII y CIII son los que presentan mayores complicaciones por su ubicación anatómica, que pueden tener características favorables o desfavorables.

lida y colaboradores ${ }^{4}$ en el 2005 mencionan que un factor que influye en que la fractura sea desfavorable es la presencia del tercer molar incluido en el trazo de fractura en el ángulo mandibular, pero no mencionan si la posición del tercer molar influye en la desfavorabilidad; su estudio se basó en la evaluación de radiografías panorámicas. Subhashraj ${ }^{5}$ en el 2009 muestra la controversia de conservar o no el tercer molar en trazo de fractura. En el estudio se pudo observar que no siempre se amerita su extracción, así como también esto puede llegar a ser un causal de complicaciones postoperatorias. Realizar una evaluación tomográfica en su corte sagital puede aportar información de la relación de la fractura con el espacio del ligamento periodontal para determinar si es una fractura expuesta. Ma'aita, ${ }^{6}$ lida $^{4}$ y Giovacchini7 indicaron que las fracturas del ángulo mandibular que ellos observaron fueron con mayor frecuencia las de aquellos pacientes con sus terceros molares con la posición C (76\%) y nivel III (85\%). En los resultados obtenidos de nuestro estudio, se observó que los terceros molares en la posición «Al» fueron más frecuentes en las fracturas de ángulo mandibular con un $31.10 \%$. Lo cual no coincide con lo antes mencionado en la literatura revisada.

De acuerdo con Ellis, ${ }^{1}$ se deduce que la correcta reposición de los fragmentos de la fractura se realiza más rápido y fácil si el diente en la línea de fractura se maneja de manera conservadora, y no se hace una evaluación de imagen en 3D de la línea de fractura y su relación con el tercer molar. Almendros ${ }^{8}$ y Escoda ${ }^{9}$ en 2008 enfatizan que al extraer el tercer molar la fractura del ángulo mandibular se desplazaría y se debería considerar si la misma pieza está impidiendo la reducción de la fractura. En su investigación no se evaluó el grado de desplazamiento ni la posición más frecuente del tercer molar presente en el trazo de fractura para evaluar la relación entre ambos conceptos explicados. Subhashraj ${ }^{5}$ en su artículo publicado en 2009 menciona que el riesgo de fracturas angulares es mayor para los terceros molares posicionados superficialmente, y disminuye para impactaciones más profundas. Esto concuerda con nuestro estudio, en donde el tercer molar Al fue más frecuente en relación con la fractura de ángulo mandibular.

James C. Fuselier ${ }^{10}$ en su estudio descriptivo con respecto a que el tercer molar aumente el riesgo de las fracturas de ángulo mandibular, en donde evaluó 1,271 pacientes, observó una edad media afectada de 30.8 años; y en lo que respecta a nuestro publicación, la población adulta joven que fue afectada

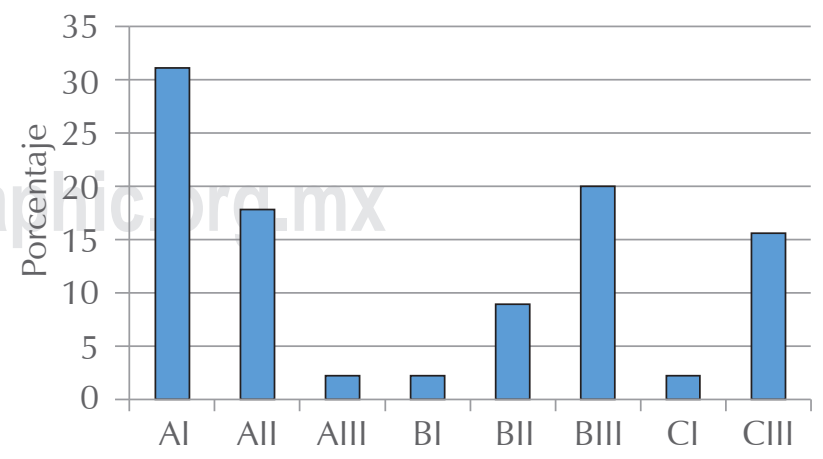

Figura 3: Porcentaje de frecuencia de la posición del tercer molar de acuerdo con la clasificación de Pell y Gregory en las fracturas de ángulo mandibular.

Fuente: Elaboración propia a través de Microsoft Excel (2017). 
estuvo en un rango de 20-29 años, observándose una diferencia mínima entre ambos resultados.

Giovacchini y colaboradores ${ }^{7}$ realizaron un metaanálisis sobre la asociación entre la fractura del ángulo mandibular y el tercer molar, en donde incluyeron siete estudios en su revisión, concluyendo que la

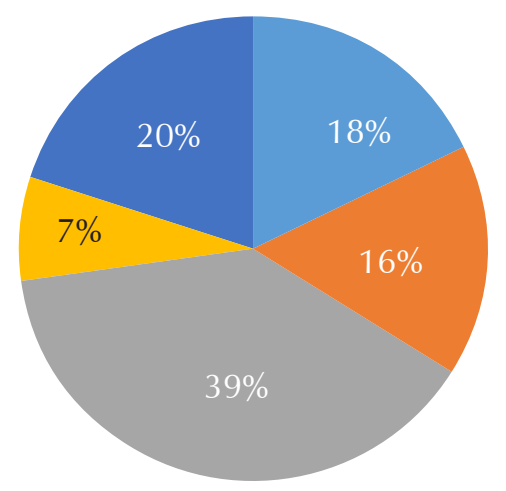

Muy favorable: $0 \mathrm{~mm}$ de desplazamiento.

Medio favorable: 3-4 mm de desplazamiento.

Poco favorable: 1-2 $\mathrm{mm}$ de desplazamiento.

Desfavorable: 5-6 mm de desplazamiento.

Muy desfavorable: $>6 \mathrm{~mm}$ de desplazamiento.

Figura 4: Desfavorabilidad en porcentajes según el grado de desplazamiento de las fracturas de ángulo mandibular con tercer molar en trazo de fractura.

Fuente: Elaboración propia a través de Microsoft Excel (2017). posición del tercer molar pareció influir en la fractura del ángulo mandibular, especialmente en las clases C, clases II y clases III, lo cual no concuerda con nuestros resultados de estudio, y que no se toma en cuenta la relación del desplazamiento de la fractura con la posición del tercer molar.

El objetivo principal de cualquier estudio es recopilar datos que tendrían un impacto positivo en el diagnóstico, el tratamiento y la prevención de una enfermedad. En la revisión de la literatura que se realizó no se menciona el uso de la tomografía asistida por computador como un instrumento de evaluación del grado de desplazamiento de la fractura. Para evaluar el grado de desplazamiento de los segmentos óseos mandibulares y la posición del tercer molar en los cortes sagitales y coronales, se tomó en consideración el techo del conducto dentario inferior.

\section{CONCLUSIÓN}

En esta investigación se observó que en las fracturas de ángulo mandibular y su relación con el tercer molar inferior según la clasificación de Pell y Gregory en trazo de fractura, el desplazamiento no está condicionado por la posición del tercer molar inferior, por lo que se descarta que la presencia del tercer molar podría jugar un papel importante que permitiera o no el desplazamiento de la fractura de ángulo mandibular, ya sea favorable o desfavorable en los 45 casos estudiados.

Tabla 2: Relación entre el desplazamiento y la desfavorabilidad con la clasificación de Pell y Gregory.

\begin{tabular}{|c|c|c|c|c|c|c|c|c|c|c|c|c|}
\hline \multirow[b]{3}{*}{ Clasificación } & \multicolumn{12}{|c|}{ Desfavorabilidad } \\
\hline & \multicolumn{2}{|c|}{1} & \multicolumn{2}{|c|}{2} & \multicolumn{2}{|c|}{3} & \multicolumn{2}{|c|}{4} & \multicolumn{2}{|c|}{5} & \multicolumn{2}{|c|}{ Total } \\
\hline & $\mathrm{F}$ & $\mathrm{Fr}$ & $\mathrm{F}$ & $\mathrm{Fr}$ & $\mathrm{F}$ & $\mathrm{Fr}$ & $\mathrm{F}$ & $\mathrm{Fr}$ & $\mathrm{F}$ & $\mathrm{Fr}$ & $\mathrm{F}$ & $\mathrm{Fr}$ \\
\hline $\mathrm{Al}$ & 4 & 0.500 & 4 & 0.571 & 3 & 0.167 & 1 & 0.333 & 2 & 0.222 & 14 & 1.794 \\
\hline All & 0 & 0.000 & 0 & 0.000 & 5 & 0.278 & 1 & 0.333 & 2 & 0.222 & 8 & 0.833 \\
\hline AIII & 0 & 0.000 & 0 & 0.000 & 1 & 0.056 & 0 & 0.000 & 0 & 0.000 & 1 & 0.056 \\
\hline $\mathrm{BI}$ & 0 & 0.000 & 1 & 0.143 & 0 & 0.000 & 0 & 0.000 & 0 & 0.000 & 1 & 0.143 \\
\hline BII & 1 & 0.125 & 0 & 0.000 & 1 & 0.056 & 0 & 0.000 & 2 & 0.222 & 4 & 0.403 \\
\hline BIII & 1 & 0.125 & 1 & 0.143 & 4 & 0.222 & 1 & 0.333 & 2 & 0.222 & 9 & 1.046 \\
\hline $\mathrm{Cl}$ & 0 & 0.000 & 1 & 0.143 & 0 & 0.000 & 0 & 0.000 & 0 & 0.000 & 1 & 0.143 \\
\hline CIII & 2 & 0.250 & 0 & 0.000 & 4 & 0.222 & 0 & 0.000 & 1 & 0.111 & 7 & 0.583 \\
\hline Total & 8 & 1.000 & 7 & 1.000 & 18 & 1.000 & 3 & 1.000 & 9 & 1.000 & 45 & 5.000 \\
\hline
\end{tabular}

Muy favorable: $0 \mathrm{~mm}$ de desplazamiento; Medio favorable: 3-4 mm de desplazamiento; Poco favorable: 1-2 mm de desplazamiento; Desfavorable: 5-6 mm de desplazamiento; Muy desfavorable: $>6 \mathrm{~mm}$ de desplazamiento.

$\mathrm{F}=$ Frecuencia; $\mathrm{Fr}=$ Frecuencia relativa.

Fuente: Elaboración propia a través de Microsoft Excel (2017). 
Tabla 3: Relación entre la desfavorabilidad y la edad.

\begin{tabular}{|c|c|c|c|c|c|c|c|c|c|c|c|}
\hline \multirow[b]{2}{*}{ Edad } & \multicolumn{2}{|c|}{1} & \multicolumn{2}{|c|}{2} & \multicolumn{2}{|c|}{3} & \multicolumn{2}{|c|}{4} & \multicolumn{2}{|c|}{5} & \multirow[b]{2}{*}{ Total } \\
\hline & $\mathrm{F}$ & $\mathrm{Fr}$ & $\mathrm{F}$ & $\mathrm{Fr}$ & $\mathrm{F}$ & $\mathrm{Fr}$ & $\mathrm{F}$ & $\mathrm{Fr}$ & $\mathrm{F}$ & $\mathrm{Fr}$ & \\
\hline 18 & 0 & 0.000 & 1 & 0.143 & 2 & 0.1111 & 0 & 0.00 & 0 & 0.0 & 3 \\
\hline 19 & 1 & 0.125 & 0 & 0.000 & 0 & 0.0000 & 1 & 0.33 & 1 & 0.1 & 3 \\
\hline 21 & 2 & 0.250 & 1 & 0.143 & 1 & 0.0556 & 0 & 0.00 & 0 & 0.0 & 4 \\
\hline 22 & 1 & 0.125 & 1 & 0.143 & 2 & 0.1111 & 0 & 0.00 & 1 & 0.1 & 5 \\
\hline 23 & 0 & 0.000 & 0 & 0.000 & 1 & 0.0556 & 0 & 0.00 & 0 & 0.0 & 1 \\
\hline 24 & 2 & 0.250 & 1 & 0.143 & 2 & 0.1111 & 0 & 0.00 & 1 & 0.1 & 6 \\
\hline 25 & 1 & 0.125 & 1 & 0.143 & 1 & 0.0556 & 0 & 0.00 & 1 & 0.1 & 4 \\
\hline 26 & 0 & 0.000 & 0 & 0.000 & 3 & 0.1667 & 0 & 0.00 & 0 & 0.0 & 3 \\
\hline 28 & 0 & 0.000 & 0 & 0.000 & 2 & 0.1111 & 1 & 0.33 & 0 & 0.0 & 3 \\
\hline 29 & 0 & 0.000 & 0 & 0.000 & 1 & 0.0556 & 0 & 0.00 & 0 & 0.0 & 1 \\
\hline 30 & 0 & 0.000 & 0 & 0.000 & 1 & 0.0556 & 0 & 0.00 & 0 & 0.0 & 1 \\
\hline 31 & 0 & 0.000 & 0 & 0.000 & 0 & 0.0000 & 1 & 0.33 & 0 & 0.0 & 1 \\
\hline 32 & 0 & 0.000 & 2 & 0.286 & 0 & 0.0000 & 0 & 0.00 & 0 & 0.0 & 2 \\
\hline 33 & 0 & 0.000 & 0 & 0.000 & 1 & 0.0556 & 0 & 0.00 & 1 & 0.1 & 2 \\
\hline 35 & 0 & 0.000 & 0 & 0.000 & 0 & 0.0000 & 0 & 0.00 & 2 & 0.2 & 2 \\
\hline 36 & 0 & 0.000 & 0 & 0.000 & 0 & 0.0000 & 0 & 0.00 & 1 & 0.1 & 1 \\
\hline 42 & 1 & 0.125 & 0 & 0.000 & 0 & 0.0000 & 0 & 0.00 & 0 & 0.0 & 1 \\
\hline 44 & 0 & 0.000 & 0 & 0.000 & 0 & 0.0000 & 0 & 0.00 & 1 & 0.1 & 1 \\
\hline 47 & 0 & 0.000 & 0 & 0.000 & 1 & 0.0556 & 0 & 0.00 & 0 & 0.0 & 1 \\
\hline Total & 8 & 1.000 & 7 & 1.000 & 18 & 1.0000 & 3 & 1.00 & 9 & 1.0 & 45 \\
\hline
\end{tabular}

Muy favorable: $0 \mathrm{~mm}$ de desplazamiento; Medio favorable: 3-4 mm de desplazamiento; Poco favorable: 1-2 mm de desplazamiento; Desfavorable: 5-6 mm de desplazamiento; Muy desfavorable: $>6 \mathrm{~mm}$ de desplazamiento.

$\mathrm{F}=$ Frecuencia; $\mathrm{Fr}=$ Frecuencia relativa.

Fuente: Elaboración propia a través de Microsoft Excel (2017).

Se observó que es mayor la frecuencia del tercer molar Al, en relación con las fracturas de ángulo mandibular que las demás posiciones del tercer molar.

La edad promedio más frecuente de los pacientes con fracturas de ángulo mandibular con el tercer molar en trazo de fractura fue de 24 años; conociéndose así que la población adulta joven que se presentó al Departamento de Estomatología del área de Cirugía Oral y Maxilofacial del Hospital Roosevelt es la más propensa a este tipo de fracturas con la presencia del tercer molar inferior en una posición Al.

Se registró que la mayor frecuencia de desfavorabilidad de las fracturas por su desplazamiento es el $33 \%$, con un resultado muy desfavorable mayor de $6 \mathrm{~mm}$ y con uno de menor frecuencia muy favorable del $7 \%$, que es igual a un desplazamiento de $0 \mathrm{~mm}$.

En este estudio se da a conocer la aportación de una nueva clasificación para evaluar el desplazamiento de los fragmentos óseos de la fractura de ángulo mandibular, en el que se utilizó la tomografía asistida por computador (TAC) para medir la distancia del techo del conducto dentario inferior, presente en cada fragmento de la fractura, lo cual es una constante anatómica en cada individuo.

Recomendaciones: se deben considerar diferentes tipos de clasificación de los terceros molares inferiores en relación con la dirección del trazo de fractura de ángulo mandibular y su desplazamiento, ya que en la evaluación de los estudios tomográficos, se observó que el tercer molar inferior en una posición horizontal con un trazo de fractura desfavorable no presentó un desplazamiento muy desfavorable considerando las medidas que se implementaron en nuestro estudio.

Se recomienda hacer un estudio comparativo entre dos poblaciones, una con la presencia del tercer molar inferior y otra población sin la presencia de éste en el trazo de fractura del ángulo mandibular; para tener un mayor alcance en el estudio se debería tomar en cuenta la escala para la evaluación del desplazamiento que se creó en nuestro estudio. 


\section{REFERENCIAS}

1. Ellis $E$ 3rd. Treatment of mandibular angle fractures using the $A O$ reconstruction plate. J Oral Maxillofac Surg. 1993; 51 (3): 250-254; discussion 255.

2. Pérez CG, Valencia GLC, Hernández OR, Pacheco LR, Valdivieso SA. Complicaciones en el manejo de fracturas de ángulo mandibular. Cir Plast. 2017; 27 (1): 5-9.

3. Pell GJ, Gregory BT. Impactado terceros molares mandibulares clasificación y técnica modificada para su eliminación. Dent Dig. 1933; 39: 330-338.

4. lida S, Hassfeld S, Reuther T, Nomura K, Mühling J. Relationship between the risk of mandibular angle fractures and the status of incompletely erupted mandibular third molars. J Craniomaxillofac Surg. 2005; 33 (3): 158-163.

5. Subhashraj K. Un estudio sobre el impacto de los terceros molares mandibulares en fracturas del ángulo. J Surg Oral Maxillofac. 2009; 67 (5): 968-972.
6. Ma'aita J, Alwrikat A. Is the mandibular third molar a risk factor for mandibular angle fracture? Oral Surg Oral Med Oral Pathol Oral Radiol Endod. 2000; 89 (2): 143-146.

7. Giovacchini F, Paradiso D, Bensi C, Belli S, Lomurno G, Tullio A. Association between third molar and mandibular angle fracture: a systematic review and meta-analysis. J Craniomaxillofac Surg. 2018; 46 (4): 558-565.

8. Almendros-Marqués N, Alaejos-Algarra E, Quinteros-Borgarello M, Berini-Aytés L, Gay-Escoda C. Factors influencing the prophylactic removal of asymptomatic impacted lower third molars. Int J Oral Maxillofac Surg. 2008; 37 (1): 29-35.

9. Escoda C, Piñera PM, Velasco VV, Berini AL. Cordales incluidos. Patología, clínica y tratamiento del tercer molar incluido. En: Escoda C, Berini AL. Tratado de cirugía bucal. Tomo I. Madrid, España: Ergón; 2004. pp. 355-356.

10. Fuselier JC, Ellis EE 3rd, Dodson TB. Do mandibular third molars alter the risk of angle fracture? J Oral Maxillofac Surg. 2002; 60 (5): 514-518. 